COMMENT. Since the first report of the effectiveness of ACTH by Sorel et al in 1958, the optimal treatment for infantile spasms is still unclear. ACTH is generally considered superior, and the use of prednisolone in the above study may account for some differences in response. Also, lack of EEG monitoring may have altered the observed response rates. For example, in contrast to the present report, previous studies have shown that the earlier the treatment, younger the patient, and higher the developmental quotient, the better the seizure and EEG outcome. (Millichap, Bickford. JAMA 1962;182:523-527; Koo et al. Neurology 1993;43:2322-2327).

In agreement with previous studies, the Great Ormond Street Hospital experience shows that cryptogenic in contrast to symptomatic spasms have $100 \%$ response to treatment and a good neurodevelopmental outcome. The authors conclude that steroids are the initial treatment of choice for infantile spasms, except for patients with tuberous sclerosis who respond to vigabatrin. That spasms are controlled faster with steroids than vigabatrin is a potential advantage, if the length of exposure to seizures and EEG hypsarrhythmia predicts cognitive outcome. Relapse rates with steroids or vigabatrin are similar. Combination, vigabatrin and steroid, a potential second-line therapy is not more effective than steroid or vigabatrin alone, but a larger controlled study may be indicated.

Add-on ketogenic diet. Short-term use (8 months) of the ketogenic diet 3:1 ratio as add-on treatment was successful in control of infantile spasms and hypsarrhythmia in 14 of 16 patients, without the growth disturbance that occurs with the traditional longterm (2 years) ketogenic diet. (Kang H-C, et al. Epilepsia 2011;52(4):781-787).

\title{
BRAIN ALKALOSIS AND BIRTH ASPHYXIA SEIZURES
}

Researchers at University of Helsinki, Finland, studied the causal mechanisms of seizures associated with birth asphyxia using 6 day-old rat pups exposed for 1 hour to hypercapnia ( $20 \% \mathrm{CO} 2$ in inhaled gas), hypoxia ( $9 \% \mathrm{O} 2)$, or both (asphyxic conditions). Normocapnia was restored gradually using CO2 levels of $10 \%$ and $5 \%$. Loss of righting reflex was used to indicate the severest expression of neonatal seizure and to quantify the seizure burden during 2-hour observation following asphyxia. Intracranial EEG was also utilized. The effect of postexposure changes in brain $\mathrm{pH}$ on seizure burden was assessed after restoration of normoxia and normocapnia. Hypercapnia or hypoxia alone had little effect on seizure burden, but rapid recovery from asphyxia was followed by a large seizure burden that paralleled a rise in brain $\mathrm{pH}$, but no change in brain oxygenation. The seizure burden and alkaline shift in brain $\mathrm{pH}$ were strongly suppressed by a graded restoration of normocapnia after asphyxia. Preapplication of N-methyl-isobutylamiloride, an inhibitor of $\mathrm{Na}+/ \mathrm{H}+$ exchanger, blocked seizures completely. Brain alkalosis after recovery from birth asphyxia has a key role in the triggering of seizures. The rapid restoration of normocapnia in the immediate postasphyxia period should be avoided and a graded restoration of brain $\mathrm{pH}$ implemented. (Helmy MM, Tolner EA, Vanhatalo S, Voipio J, Kaila K. Brain alkalosis causes birth asphyxia seizures, suggesting therapeutic strategy. Ann Neurol April 2011;69:493-500). (Respond: Dr Kaila, Dept Biosciences, PO Box 65, 00014 University of Helsinki, Helsinki, Finland. E-mail: kai.kaila@helsinki.fi). 
COMMENT. Seizures in the neonate with hypoxic-ischemic encephalopathy are often subtle, characterized by swimming, rowing movements, apneic spells, opisthotonus, or clonic movements. (Volpe JJ. Observing the infant in the early hours after asphyxia. In Gluck L, ed. Intrauterine asphyxia and the developing fetal brain. Chicago, Year Book Medical Publ, 1977).

In newborn rats, seizures induced by electroshock vary with strength of current and with age. In 10-day-old rats, the animal exhibits hyperkinetic behavior, breaststroke swimming movements, and transient opisthotonus. At 20 days, tremor of head, and catatonic posture; and at 30 days, the seizure is a generalized symmetrical clonus with loss of posture. A stronger current is required to induce a maximal tonic clonic seizure in rats older than 15-20 days. In rats aged 1 to 15 days, a maximal tonic seizure cannot be elicited. The increased severity of seizure response with increase in age is correlated with development of brain carbonic anhydrase. (Millichap JG. Development of seizure patterns in newborn animals. Significance of brain carbonic anhydrase. Proc Soc Exptl Biol Med 1957;96:125). If slightly older rat pups ( $>6$ days) had been employed in the Helsinki experiment, a more definitive seizure endpoint might have been elicited. The following clinical report supports the association of PCO2 and outcome of HIE in neonates, but raises questions regarding a seizure as the measure of brain damage and disability.

Pappas A and colleagues (J Pediatr May 2011;158:752-758) in the Eunice Kennedy Shriver NIH Neonatal Research Network report that infants exposed to low $\mathrm{PCO} 2$ concentrations within the first 16 hours of life during whole body cooling were at increase risk of death/disability. Disability was directly related to the degree and severity of hypocarbia. Both minimum PCO2 and cumulative PCO2 $<35 \mathrm{mmHg}$ were associated with poor outcome at 18-22 months of age. The occurrence of clinical seizures was not significantly associated with death or disability. The infants had received ventilatory resuscitation or assistance at birth, and this may have contributed to hypocarbia. Further investigation will determine whether hypocarbia is a modifiable risk factor or simply a marker of poor outcome. Clyman RI, in an editorial (Jrnl Pediatr 2011;158:A2), asks "Should cooled infants be started on lower initial ventilator settings or weaned more rapidly?" "Will avoidance of hypocarbia improve outcome?"

\section{ANTICONVULSANT DRUGS}

\section{FOLIC ACID SUPPLEMENT PREVENTS PHENYTOIN-INDUCED GINGIVAL HYPERPLASIA}

Researchers at the Division of Pediatric Neurology, Neurosciences Centre, New Delhi, India, performed a randomized, double-blind, placebo-controlled 6-month trial of oral folic acid supplement $(0.5 \mathrm{mg}$ /day) in children aged $6-15$ years treated with phenytoin for epilepsy. Of 62 patients who received folic acid supplement, $21 \%$ developed gingival hyperplasia whereas of 58 receiving placebo, $88 \%$ developed hyperplasia $(\mathrm{p}<0.001)$. Risk reduction of phenytoin-induced gingival hyperplasia by folic acid was $67 \%$. The mean phenytoin dose was similar $(6.8$ and $7.0 \mathrm{mg} / \mathrm{kg} / \mathrm{day}$ respectively) in folic acid and placebo groups at the end of the study. Mean serum levels of phenytoin were therapeutic $(13 \mathrm{mcg} / \mathrm{ml})$ in both groups. Seizures were symptomatic, 